\title{
Neutrophilic reversible allograft dysfunction (NRAD) and restrictive allograft syndrome (RAS)
}

Stijn E Verleden ${ }^{1}$, Elly Vandermeulen ${ }^{1}$, Robin Vos ${ }^{1}$, David Ruttens ${ }^{1}$, Lieven J Dupont ${ }^{1}$, Dirk E Van Raemdonck ${ }^{1}$, Bart M Vanaudenaerde ${ }^{1}$ and Geert M Verleden ${ }^{1}$,

${ }^{1}$ Lung Transplantation Unit, KULeuven and University Hospital Gasthuisberg, Leuven, Belgium

GMV is holder of GSK chair in respiratory pharmacology at the KULeuven, and supported by Research Foundation Flanders (FWO): G.0723.10, G.0705.12 and G.0679.12 and 'Onderzoeksfonds K.U.Leuven (OT/10/050). BMV, DEVR are senior research fellows of FWO. None of the funding sources have a role in study design, conduction and reporting of this study.

Address for correspondence: $\quad$ Prof G.M. Verleden Lung Transplantation Unit

49 Herestraat, B-3000 Leuven, Belgium Tel: + 3216346805 Fax: + 3216346803

E-mail: geert.verleden@uzleuven.be 


\section{Summary}

Lung transplantation is currently considered as an ultimate live-saving treatment for selected patients suffering from end-stage pulmonary disease. Long-term survival, however, is hampered by chronic rejection, or chronic lung allograft dysfunction (CLAD). Recently, various phenotypes within CLAD have been identified, challenging the established clinical definition of Bronchiolitis Obliterans Syndrome (BOS). Some patients with presumed BOS for instance, demonstrate an important improvement in $\mathrm{FEV}_{1}$ after treatment with azithromycin. These patients are characterized by the presence of excess ( $\geq 15 \%)$ BAL neutrophils, in absence of concurrent infection. This phenotype of CLAD has been redefined as neutrophilic reversible allograft dysfunction (NRAD) and these patients generally have a very good prognosis after diagnosis. Another group of patients with CLAD develop a restrictive rather than an obstructive pulmonary function defect (defined as a decline in total lung capacity of at least $10 \%$ ) and demonstrate persistent interstitial and ground glass opacities on chest CT scan. This phenotype is called restrictive allograft syndrome (RAS) and patients with RAS have a much worse prognosis after diagnosis. In this review, we will further discuss both of these CLAD phenotypes that do not fit the classical definition of BOS. Potential pathophysiological mechanisms, etiology, diagnosis, prognosis and treatment will be discussed. 


\section{Introduction}

Lung transplantation (LTX) has become an established treatment option for patients with end-stage pulmonary diseases like COPD, cystic fibrosis, pulmonary fibrosis, pulmonary arterial hypertension, ... Survival after LTx, however, remains rather poor as only $55 \%$ of patients survive more than 5 years according to the registry of the International Society for Heart and lung Transplantation (ISHLT) database (1). Early post-operative complications include primary graft dysfunction (2), infections, suture stenosis (3), acute rejection (4) and lymphocytic bronchiolitis (LB) (5). Long-term survival is limited by infections and the development of chronic rejection or chronic lung allograft dysfunction (CLAD), which may clinically manifest as Bronchiolitis Obliterans Syndrome (BOS), an obstructive decline in $\mathrm{FEV}_{1}$ of at least $20 \%$ compared to the best post-operative value in the absence of other identifiable factors like acute rejection, suture stenosis, infection, ... It was the general believe that BOS was characterized by: A/ neutrophilic airway inflammation, B/ largely irreversible and persistent obstructive pulmonary function decline, and $\mathrm{C}$ / a fibroproliferative narrowing of the bronchioles (6). As diagnostic yield of transbronchial biopsies is very low (7), a decline in $\mathrm{FEV}_{1}$ of at least $20 \%$ is regarded as the hallmark of chronic rejection in the absence of other identifiable causes. There is now accumulating evidence that not every persistent decline in $\mathrm{FEV}_{1}$ is compatible with this definition and as a consequence all over the world the term CLAD has been introduced, although up to now this term has not clearly been defined. Nevertheless, there is growing awareness that this terminology may be better to identify a chronic, persistent decline in FEV ${ }_{1}$ after LTx. As a consequence, this review will focus on 2 different non-BOS phenotypes of CLAD. More specifically, we will focus on neutrophilic reversible allograft dysfunction (NRAD), characterized by an increase in $\mathrm{FEV}_{1}$ of at least $10 \%$ after azithromycin treatment on the one hand, and the recently identified restrictive allograft syndrome (RAS) on the other hand. 


\section{Neutrophilic reversible allograft dysfunction}

\section{Neutrophils}

Digiovine et al. were the first to demonstrate the presence of excess BAL neutrophils during chronic rejection (8), which was further corroborated by Riise et al. who demonstrated that neutrophil infiltration and activation plays a role in the development of BOS. Convincing evidence of neutrophil activation was demonstrated in BAL fluid and in transbronchial biopsies obtained from lung transplanted patients with BOS, moreover BAL fluid levels of IL-8 and neutrophils significantly increased in patients diagnosed with BOS (9). Thereafter, more and more studies corroborated the association between excess BAL neutrophilia and the development of chronic rejection and survival after lung transplantation (10-12). Moreover, the presence of BAL neutrophils at 3 months and 1 year after transplantation proved to be prognostic for the later development of BOS (13). Neutrophils were thought to be responsible for the development of obliterative bronchiolitis $(\mathrm{OB})$ via secretion of matrix metalloproteinases (14), different chemokines and growth factors and oxidative stress (15), causing damage to the respiratory epithelium, leading to an excess repair process with proliferation of fibroblasts and finally established $\mathrm{OB}$.

\section{Neomacrolides}

The introduction of the neomacrolide antibiotic azithromycin, however, has led to a complete paradigm shift. Gerhardt et al. were the first to use low-dose azithromycin ( $250 \mathrm{mg}$ three times a week) as an add-on treatment to conventional immunosuppressive therapy in six patients with BOS after LTx. FEV 1 improved in five of these six patients by a mean of $17.1 \%$ or $0.50 \mathrm{~L}$ over a 4 -month period (16). This was later on corroborated by several other groups (17-20), all reporting an increase in $\mathrm{FEV}_{1}$ in at least a subset of patients, clearly illustrating the beneficial effect of azithromycin in established BOS. However, not all studies reported a clear beneficial effect of azithromycin. Shitrit et 
al. for instance, demonstrated that the overall $F E V_{1}$ of 11 LTx patients (BOS 3: $n=1 ; B O S 2: n=6$; BOS 1: $\mathrm{n}=4$ ) had decreased by $1 \%$ over 4 months and $2 \%$ after 10 months of additional azithromycin treatment. Azithromycin therapy also did not lead to any improvement of BOS status, however, it could at least arrest the progression of the disease (21). The reason why not all patients responded to azithromycin treatment was not clear at first. In fact, several possible reasons were raised such as a change in calcineurin trough level, the initial BOS stage at the start of azithromycin treatment, colonisation of the airways with Pseudomonads,... (16-19). Later on, however, it became clear that patients who responded to azithromycin, had high BAL IL-8 levels and neutrophilia at the time of diagnosis. Moreover, there was a good correlation between the initial BAL neutrophilia and the $\mathrm{FEV}_{1}$ response after 3 months of treatment (22), a finding that was later corroborated by Gottlieb et al. (17). The observed discrepancy between responders and non-responders disclosed a dichotomy within chronic rejection after lung transplantation. The first phenotype displays a high \% of neutrophils in BAL (> 15\%), develops rather early after LTx and displays an improvement in $\mathrm{FEV}_{1}$ of at least $10 \%$ after 3 to 6 months of azithromycin treatment. This phenotype was called neutrophilic reversible allograft dysfunction (NRAD) or neutrophilic CLAD (nCLAD). This is in big contrast with the other phenotype, which lacks high BAL neutrophilia $(<15 \%)$, develops later and does not respond to azithromycin therapy and was denominated at that moment as fibroproliferative Bronchiolitis Obliterans Syndrome (fBOS) $(23,24)$. A randomized double blind placebo-controlled trial recently showed that azithromycin started at discharge from the hospital after LTx could prevent the development of CLAD. The azithromycin group also displayed a better $\mathrm{FEV}_{1}$, lower BAL neutrophils and lower systemic C-reactive protein (CRP) levels compared to the placebo group (25).

At this moment it is still unclear whether a NRAD episode any time during follow-up is a risk factor for the later development of BOS, but it has been shown that NRAD is at least a risk factor for longtime survival after LTx (26). Azithromycin is not the only macrolide used in post-transplant care, some groups prefer to use clarithromycin, which results in a similar effect in a similar way (responders vs. 
non- responders) (27). Caution is needed when using clarithromycin, as it affects the calcineurin trough levels whereas azithromycin does not $(28,29)$.

\section{Mechanisms of action of azithromycin}

We assume that both the antimicrobial and the anti-inflammatory actions contribute to the beneficial effect of neomacrolide antibiotics. The exact cellular mechanisms are beyond the scope of this review and have already extensively been described previously (30). Some aspects, however, are important to mention. A human BAL study looking at the expression of 32 different proteins in BOS, NRAD and control patients at the time of diagnosis of CLAD showed that in established NRAD patients MCP-1, RANTES, IL-1 $\beta$, IL-8 , TIMP-1, MMP-8, MMP-9, HGF, MPO and bile acid concentrations were upregulated, while only PDGF-AA was downregulated compared to control patients (31). Moreover almost all these proteins were correlated with BAL neutrophilia. None of the proteins were different between azithromycin non-responsive BOS or fBOS and control patients. This is in sharp contrast with previously published data showing that these proteins are indeed involved in the development of BOS (32-34). This further illustrates the importance for adequate phenotyping of BOS. Moreover, one should keep this in mind when looking at previous research as all this work was performed with the understanding of BOS at that time, making interpretation of these results very difficult as we now know that BOS actually represents different phenotypes. An interesting future direction of research is to see whether azithromycin is able to decrease all these proteins to levels comparable to stable patients. It has for instance recently been shown that MMP-9 gelatinase activity is increased in BAL of NRAD patients and following 3-6 months of azithromycin therapy the MMP-9 gelatinase activity significantly decreased. More importantly, these decreased levels were still higher compared to stable patients, which might indicate an ongoing matrix remodeling process, potentially leading to the later development of chronic rejection (35). An intriguing hypothesis is that IL-17, an important pro-inflammatory cytokine and indirect chemo-attractant of neutrophils, is a key 
player in NRAD. Evidence derived from either human or mice studies already implicated IL-17 in the pathogenesis of chronic rejection (36-38). In vitro data in human airway smooth muscle cells and epithelial cells shows that IL-17 can induce IL-8, a major neutrophil chemo-attractant, but more importantly that azithromycin is able to reduce the IL-17 induced IL-8 production $(39,40,40)$. Further unpublished results show that the number of IL-17 positive lymphocytes in the submucosa increase in NRAD compared to control and azithromycin non-responsive patients. After 3 to 6 months of azithromycin therapy the number of IL-17 positive cells significantly decreased to control levels.

It remains to be added that during azithromycin treatment for NRAD, there may be a recurrence of BAL neutrophilia with or without concurrent $\mathrm{FEV}_{1}$ decline, the significance and prognosis of which remain to be further elucidated.

Lymphocytic bronchiolitis and NRAD: different entities or not?

Lymphocytic bronchiolitis (LB) is characterized by a lymphocytic infiltration of the airway submucosa and the epithelium and is graded according to the extent of the infiltrate (41). LB is often accompanied by a decrease in pulmonary function (42). Glanville et al. showed that LB is a risk factor for the later development of BOS (43). Regarding treatment of LB, there is conflicting evidence showing that inhaled steroids might bring some relief. One study indeed shows an increase in $\mathrm{FEV}_{1}$ after treating LB with inhaled steroids (44), while another study could not demonstrate a beneficial effect (42).

There may actually be a lot of similarities between LB and NRAD. As described above, LB is characterized by a lymphocytic infiltrate in the airway submucosa but a study by Chambers et al. also noticed the presence of a lymphocytic infiltrate in the bronchial/bronchiolar epithelium of established BOS patients. Moreover the number of T-cells in this infiltrate correlated with the BAL neutrophilia (45). BAL neutrophilia is indeed the most typical characteristic of NRAD patients, but BAL 
neutrophilia can also be found during episodes of LB. Vos et al. indeed demonstrated that the $\%$ BAL neutrophils is significantly higher during LB compared to control patients and patients suffering from acute perivascular rejection (46). Lastly, when comparing CT scans of LB patients with azithromycin responsive patients, we observe very similar patterns: centrilobular nodules and tree in bud, which resolve after 2-6 months of azithromycin therapy (47). It is our believe that some episodes of LB, especially when accompanied by BAL neutrophilia, may result in a decline in FEV ${ }_{1}$ similar to NRAD. Moreover it seems likely that a negative score for B-grade rejection at diagnosis of NRAD is simply a matter of sampling error as biopsies are very small and can give a skewed image (48). We are currently recruiting patients with isolated LB, not yet treated with azithromycin, in an open label trial with azithromycin to establish its true effect in this situation. (ClinicalTrials.gov Identifier: NCT01109160). In figure 1, we illustrate the $\mathrm{FEV}_{1}$ evolution of a patient with biopsy-proven LB (patient 1) and one with NRAD (patient 2). Both were only treated with additional azithromycin and show a comparable increase in $\mathrm{FEV}_{1}$. Since we know that high doses of steroids hardly result in any improvement of FEV1 in patients with isolated LB (42), this particular situation may therefore represent another beneficial effect of neo-macrolides. 


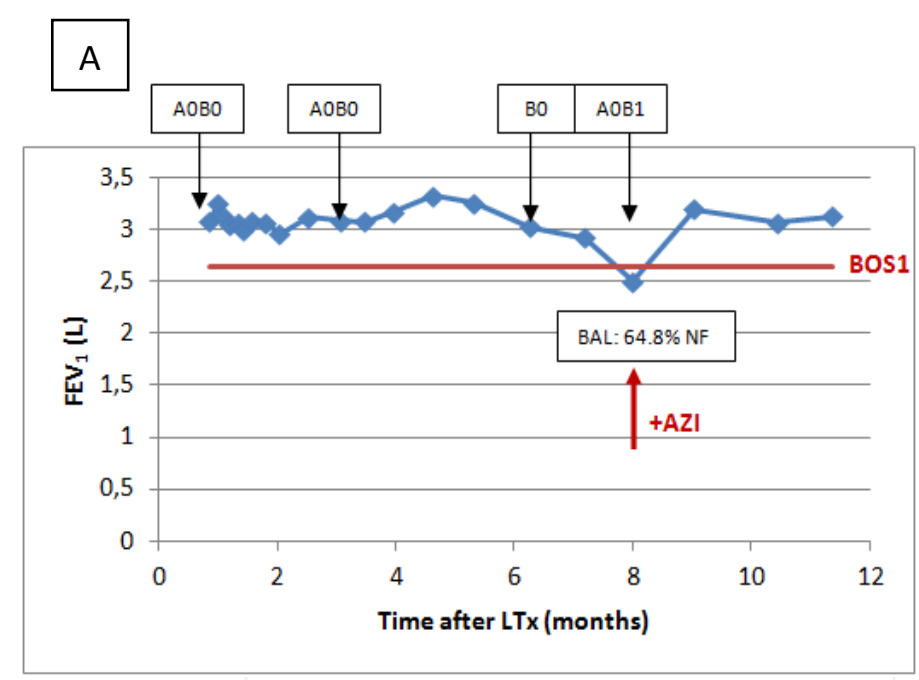

\section{B}

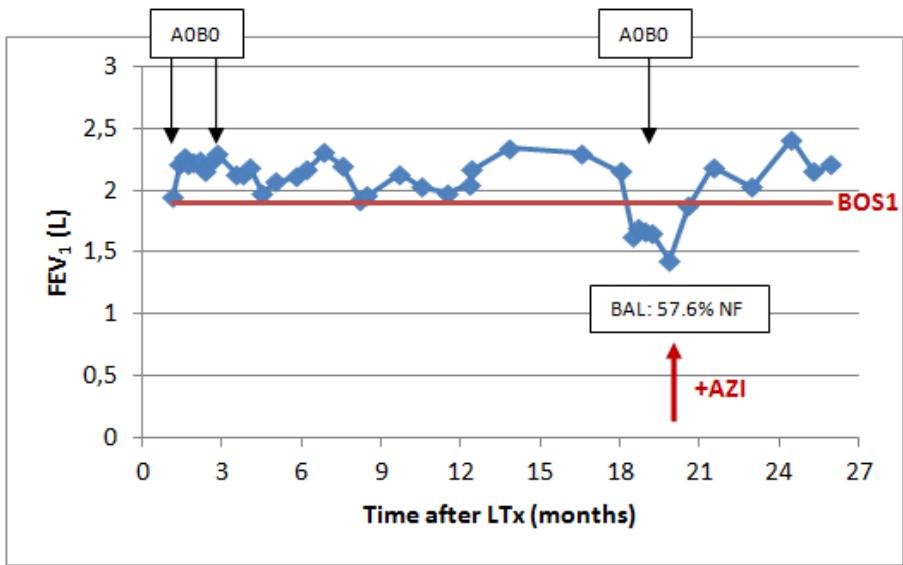

Figure 1 Legend

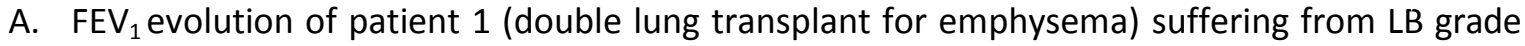
B1R with $64.8 \%$ of neutrophils in the BAL fluid. At that time there was a single $\mathrm{FEV}_{1}$ measurement reaching the threshold of BOS1. Following therapy with azithromycin (red arrow) the $\mathrm{FEV}_{1}$ fully recovered.

B. FEV ${ }_{1}$ evolution of patient 2 (double lung transplant for emphysema, suffering from NRAD. After 18 months the patients experienced a persistent decrease (at least 3 weeks) in $\mathrm{FEV}_{1}$ compatible with BOS. A biopsy at that time could not demonstrate perivascular or peribronchiolar rejection. BAL showed $57.6 \%$ neutrophils. Azithromycin was started (red arrow), leading to a complete restoration of the $\mathrm{FEV}_{1}$, resulting in $\mathrm{BOS} 0$ again. 


\section{Restrictive allograft syndrome}

\section{Diagnosis and prognosis}

Recently, the Toronto group introduced the term 'restrictive allograft syndrome' (RAS) for patients suffering from a persistent decline in $\mathrm{FEV}_{1}$ ( $>20 \%$ compared with the best postoperative values) and an associated restrictive pulmonary function defect, which they defined as a decline in total lung capacity (TLC) $>10 \%$ compared to baseline (49). As TLC is not routinely performed in most centers, Verleden et al. also used the $\mathrm{FVC}$ and $\mathrm{FEV}_{1} / \mathrm{FVC}$ ratio as a surrogate marker. If the latter remains stable while the $\mathrm{FEV}_{1}$ drops, this may also point to a restrictive pulmonary function (26). In that respect, Sato demonstrated a good correlation between $\mathrm{FEV}_{1} / \mathrm{FVC}$ ratio and TLC (49).

This syndrome develops in about $25-35 \%$ of all lung transplant patients suffering from CLAD. Moreover, these RAS patients demonstrated a lower survival rate compared with BOS patients. The median survival after diagnosis of RAS was 8 months in the Leuven cohort, while the Toronto cohort showed a median survival of 16 months, which was considerably lower compared to patients with an obstructive pulmonary function defect (hence typical BOS patients, 35 months in Leuven and 46 in Toronto) $(26,49)$. CT scan in RAS patients shows more interstitial opacities, ground glass opacities, upper lobe dominant fibrosis and honeycombing compared to patients with BOS, which show more signs of airtrapping (49).

Presently, the pathophysiological mechanisms leading to RAS remain elusive. In the Toronto cohort, 12 of 29 patients (41\%) had a positive identification of a wide range of micro-organisms in either BAL or sputum at diagnosis, pointing to a possible infectious trigger that might lead to an excessive fibrotic reaction, resulting in a lung with end-stage fibrosis (49).

Although the terminology may be new, the syndrome seems to have been described previously in autopsy studies. In a report by Martinu et al, studying 12 retransplant lungs, at least 3 patients may now be identified as having RAS with pathology reports showing interstitial fibrosis and radiology showing ground glass opacities, intralobular thickening and areas of fibrosis (50). Moreover, interstitial changes on transbronchial biopsies are already reported for a long time and seem to occur 
more frequently later on during follow-up (51). In chronic graft versus host disease with pulmonary manifestation after bone marrow or stem cell transplantation, a similar phenomenon has already been described. This syndrome is denominated as pleuroparenchymal fibro-elastosis and is a very rare complication. Patients manifested with dyspnoea, CT showed pleural thickening, interstitial fibrosis and mosaic patterns, while the pathology demonstrated obliterative bronchiolitis combined with patchy zones of intra-alveolar fibrosis $(52,53)$. Unfortunately no detailed pulmonary function tests were performed. In this small case series prognosis was also bad, similar to RAS. The similarities between RAS and pleuroparenchymal fibro-elastosis are hence very striking and might represent a similar pathophysiological mechanism. This is also noted by the Toronto group as they reported the great similarities between the 2 conditions. One of the most remarkable findings of their study is the presence of $\mathrm{OB}$ in RAS patients (14/16 patients, $87.5 \%)$, which indicates that RAS might in fact be a manifestation of chronic rejection (54). Pathological analysis of an explant lung at re-transplantation for RAS indeed shows obliteration of the airway (figure 2A) with surrounding fibrosis of the alveoli. Masson's trichrome staining is able to demonstrate accumulation of granulation tissue within the airway lumen and within the parenchyma a dens area of fibrosis is seen (figure 2B).

Further research will indicate whether the definition of RAS will stand and whether more different phenotypes of RAS will be defined. In this respect, there may indeed be differences in radiological presentations as some patients do have extensive apical pleural fibrosis, whereas others rather develop interstitial changes throughout the lung, although most apparent in the upper lobes. In this respect, the paper by Pakhale et al (48) describing upper lobe fibrosis probably represents the first clinical presentation of RAS.

BOS and RAS may also co-exist, as some patients first develop a classical BOS (obstructive decline in FEV1, no decline in TLC) and only later on develop characteristics of RAS (decline in TLC $\geq 10 \%$ ) with the appearance of interstitial infiltrates on CT scan.

Does this all represent the same manifestation of the disease or are different pathophysiological mechanisms responsible for this? Most importantly, will this have an influence on the survival of the 
patients? What about other manifestations of chronic rejection, not strictly fitting the definition of BOS or RAS such as follicular bronchiolitis (55), exudative bronchiolitis (56)? This all remains to be further investigated.

\section{RAS and Pulmonary function}

Woodrow was the first to introduce the term restrictive BOS, based on a decrease of the forced vital capacity (FVC) of at least $20 \%$ compared to baseline. This study, however, could not demonstrate a survival disadvantage for the patients suffering from a restrictive pulmonary function, although a trend was seen, probably due to exclusion of patients with persistent infiltrates, who most likely also suffered from a restrictive pulmonary function (57).

The studies, documenting and describing RAS patients after lung transplantation, used different criteria to diagnose a restrictive pulmonary function. Sato et al defined RAS, along with an FEV ${ }_{1}$ decline of at least $20 \%$, as an additional TLC decline of at least $10 \%$ compared to baseline. Verleden et al. also incorporated the tiffeneau index to describe restriction and Woodrow, as discussed above, used an FVC decline of at least $20 \%(25,45,51)$. Further research will show if there is an easier method to diagnose RAS as TLC measurements are not routinely performed in most centers, although it is advised now to do so. An easier method to diagnose RAS would surely benefit an earlier and a more accurate diagnosis. If this would prove to be ineffective, then more extensive pulmonary function testing will need to be performed more often in the routine transplant patient follow-up. Two different types of RAS patients are illustrated in figure 3. One patient shows a very fast evolution towards re-transplantation, while the other patient shows a rather slow evolution.

\section{Risk factors}

The only identified risk factor up to now for the later development of RAS is late onset diffuse alveolar damage (DAD occurring later than 3 months after LTx) as $58 \%$ of RAS patients suffered from an episode of late onset DAD (58). The problem, however, is that only approximately $30 \%$ of patients 
with chronic rejection suffer from RAS, making it very difficult to do single-center studies. Multicenter studies are necessary to accurately define risk factors for RAS. Perhaps similar risk factors as compared to BOS (A grade rejection, LB, CMV infection, pseudomonas colonization, gastrooesophageal reflux, ...) will prove to be important for the later development of RAS (59). Some new risk factors may arise such a genetic predisposition towards the development of RAS. Interesting in that perspective is the fact that a MUC5B polymorphism has been demonstrated to be an important genetic predictor for Idiopathic Pulmonary Fibrosis (IPF) (60), which may in some way resemble RAS development. This has hitherto not been investigated in the development of RAS.

Since serum KL-6 levels, a marker of fibroblast proliferation, are increased in RAS patients compared to BOS patients (61), this may strengthen this hypothesis as KL-6 is also a marker for IPF or CF, diseases characterized by interstitial fibrosis $(62,63)$. The patient cohort was, however, rather small, and future studies might be able to corroborate the potential role of this intriguing marker.

In the Toronto experience, neither age (donor/receptor), type of transplantation, original diagnosis for LTx, CMV (mis-)match and acute rejection proved to be risk factors for the later development of RAS (49).

\section{Treatment}

As the concept of RAS is rather new, no valid treatment has been proposed yet. At this moment the influence of conventional BOS treatment options like total lymphoid irradiation (64), photophoresis (65) and montelukast (66) is not clear. In our experience azithromycin does not bring relief to these patients. Perhaps we should look outside the field of lung transplantation and extend our horizon to IPF, a disease with much similarities to RAS as the etiology of the disease is unknown, radiology looks similar (consolidation zones, reticular patterns) and histology shows end-stage fibrosis. A beneficial effect of pirfenidone has been shown to slow down the decline in FVC in patients with established IPF (67). Pirfenidone suppresses TNF- $\alpha$ production and influences the production of TGF- $\beta$ (68). Moreover, in vitro studies showed that pirfenidone was able to slow down proliferation of human 
lung fibroblast cells (69). Even within the lung transplant setting, there is some evidence that pirfenidone might bring relief, although this evidence is mostly based on animal studies. Both in a murine heterotopic tracheal transplantation model $(70,71)$ and a rat orthotopic lung transplantation model, pirfenidone has been shown to reduce fibrosis (72). At this moment, however, the only treatment that can bring relief in well-selected patients is re-transplantation. Another drug that might be of help is the CD52 antagonist alemtuzumab (Campath-1H). CD52 is a protein expressed on B-cells, lymphocytes, dendritic cells and monocytes and was first used in lung transplantation to treat recurrent acute rejection episodes, which were untreatable with conventional treatment protocols (73), with promising results. A further study used alemtuzumab in treatment of both refractory acute rejection ( $n=12$ ) and bronchiolitis obliterans syndrome $(n=10)$, showing improvement in BOS stage in 4 patients, stabilization in 3 and further detoriation in the 3 other patients (74). Indications that it can be used in RAS came from 2 recent reports. Treatment with alemtuzumab in a patient suffering from pulmonary chronic graft versus host disease after bone marrow transplantation showed impressive improvement in pulmonary condition. The initial radiology, resembling RAS, shows patchy consolidation and diffuse reticulonodular areas, which completely resolved after 20 days of treatment (75). Alemtuzumab further improved lung function in 4 patients who developed interstitial lung injury with alveolar septal fibrosis, typical hallmarks of RAS patients (76).

\section{Conclusion}

Phenotyping CLAD has very important implications towards clinical practice. NRAD patients are most easily to diagnose using BAL fluid and these patients will recover following azithromycin treatment (as per definition) and have a very good prognosis. RAS patients are diagnosed using TLC and CATscans and have a much worse prognosis. Unless other treatments become available to treat RAS, this implicates that possible re-transplantation should be considered quickly after diagnosis as prognosis is bad. Moreover phenotyping CLAD also has important scientific implications as most previous studies used a pool of patients suffering from BOS/CLAD whereas it is now presumed that each 
phenotype has its own characteristics and possibly different risk factors, which implicates that accurate phenotyping is necessary for valid scientific research. An overview of different characteristics representing the different CLAD phenotypes is given in table 1 . Only by adequate phenotyping patients, a better long term survival can be achieved. The future will probably lie in an individualized therapy depending on the type of CLAD that the patient is suffering from. This is probably the only hope to win the battle against chronic rejection after lung-transplantation and to finally have a long term survival that matches other solid-organ transplants. 


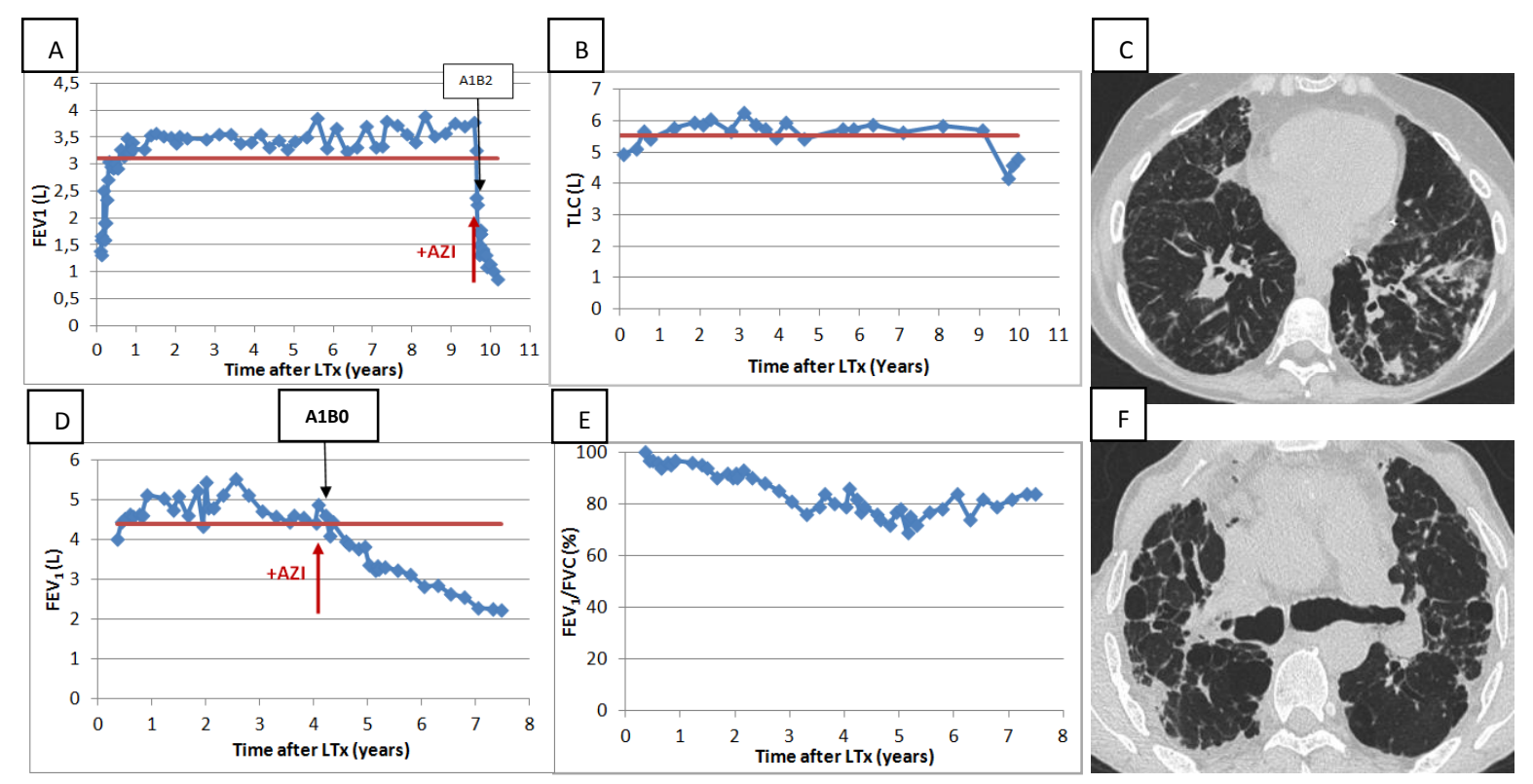

Figure 2

a) $\mathrm{FEV}_{1}$ evolution of patient 1 with RAS. 9 years after initial double lung transplantation for cystic fibrosis, the patient develops a very rapid irreversible decline in FEV ${ }_{1}$. Red line indicates a decline of $20 \%$.

b) TLC evolution of RAS patient 1 . When the $\mathrm{FEV}_{1}$ starts declining a decrease in TLC $>10 \%$ was observed compatible with the definition of RAS. Red line indicates a decline in TLC of $10 \%$.

c) CAT scan of patient 1 at diagnosis of RAS. CAT shows ground glass opacities with accompanying zones of consolidation.

d) FEV $_{1}$ evolution of patient 2 with RAS. 4 years after initial double lung transplantation for $\alpha 1-$ anti trypsin deficiency, the patient develops a slow decline in FEV 1 . Red line indicates a decline of $20 \%$.

e) Tiffeneau index evolution of patient 2 as no TLC measurements before development of RAS are available. As the $\mathrm{FEV}_{1}$ declines, the Tiffeneau index does not drop below the baseline of 0.7 compatible with a restrictive pulmonary function decline.

f) CAT scan of patient 2, 2 years after diagnosis of RAS, showing severe pleural and septal thickening. 
Figure 3

A) H\&E staining of an explanted lung of a RAS patient at re-transplantation. A dense accumulation of granulation tissue is seen within the airway lumen. The airway parenchyma is partially fibrosed resulting in very dense tissue. Black arrow points to the airway wall.

B) Masson- trichrome staining on an explanted lung of a RAS patient at re-transplantation. The airway lumen is filled with additional collagen. Within the airway parenchyma a dense collagen positive infiltrate is seen. Black arrow points to the airway wall, dashed arrow points to fibrosis within the parenchyma.
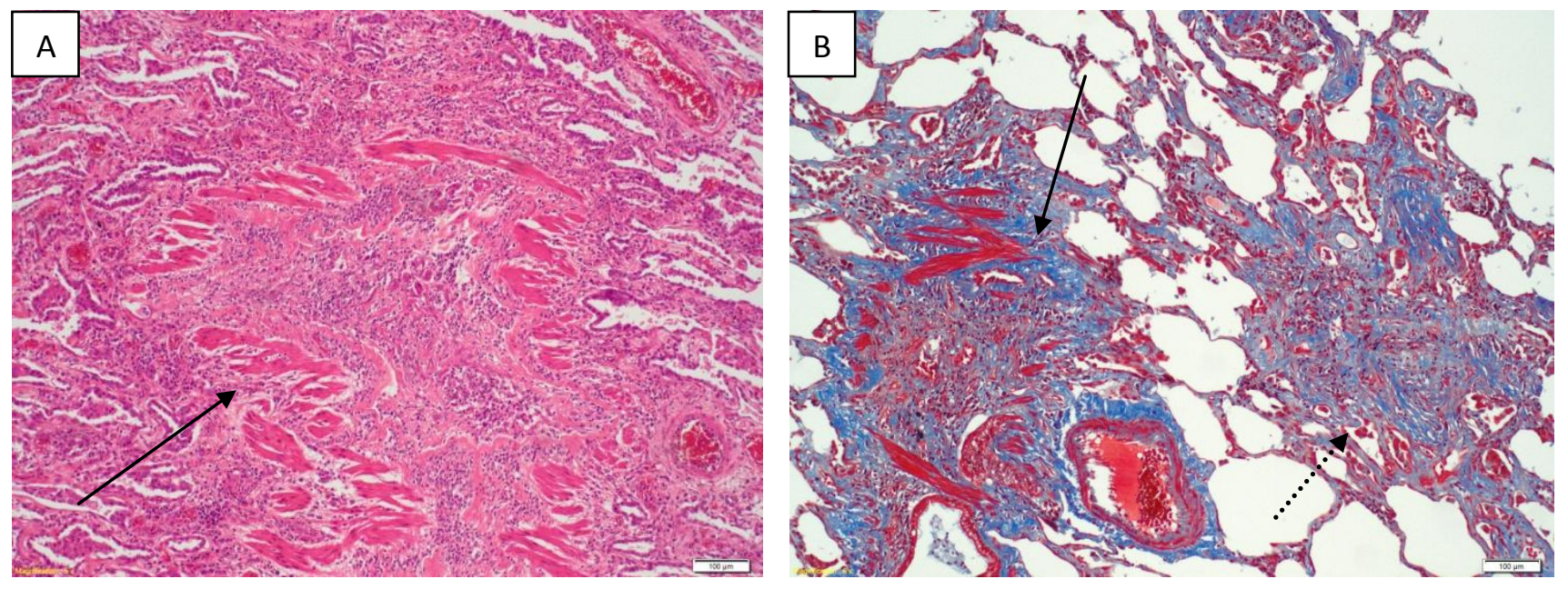


\begin{tabular}{|c|c|c|c|}
\hline & NRAD & BOS & RAS \\
\hline Inflammation (BAL) & neutrophilic airway inflammation & no airway inflammation & airway inflammation? \\
\hline Clinically & coarse crackles and increased sputum production & no coarse crackles, minimal sputum & Minimal coarse crackles \\
\hline Time of onset & mostly early onset after transplantation & onset late after transplantation & Onset late after transplantation \\
\hline Survival & very good & fairly good & bad \\
\hline Histology & $\begin{array}{l}\text { initially inflammatory } \\
\text { ends up in pure fibrosis }\end{array}$ & OB lesions, healthy parenchyma & $\begin{array}{l}\text { parenchymal fibrosis, } \\
\text { thickened septa and pleura }\end{array}$ \\
\hline Radiology & $\begin{array}{l}\text { bronchiectasis, airway wall thickening, } \\
\text { mucus plugging, centrilobular nodules }\end{array}$ & air trapping and mosaic attenuation & $\begin{array}{c}\text { consolidation, reticular pattern, } \\
\text { persistent infiltrate }\end{array}$ \\
\hline Azithromycin & reversible (effective) & irreversible (ineffective) & irreversible \\
\hline Pulmonary function & obstructive & obstructive & $\begin{array}{l}\text { restrictive (TLC decline }>10 \% \text { ), } \\
\text { increasing tiffeneau index }\end{array}$ \\
\hline
\end{tabular}

Table 1: proposal of different characteristics in NRAD, BOS and RAS patients (partially adapted from (23)) 
1. Trulock EP, Christie JD, Edwards LB et al. Registry of the International Society for Heart and Lung Transplantation: twenty-fourth official adult lung and heart-lung transplantation report2007. J Heart Lung Transplant 2007;26: 782-795.

2. Lee JC, Christie JD, Keshavjee S. Primary graft dysfunction: definition, risk factors, short- and long-term outcomes. Semin Respir Crit Care Med 2010;31: 161-171.

3. Colquhoun IW, Gascoigne AD, Au J, Corris PA, Hilton CJ, Dark JH. Airway complications after pulmonary transplantation. Ann Thorac Surg 1994;57: 141-145.

4. Davis WA, Finlen Copeland CA, Todd JL, Snyder LD, Martissa JA, Palmer SM. Spirometrically significant acute rejection increases the risk for BOS and death after lung transplantation. Am J Transplant 2012;12: 745-752.

5. Burton CM, Iversen M, Scheike T, Carlsen J, Andersen CB. Is lymphocytic bronchiolitis a marker of acute rejection? An analysis of 2,697 transbronchial biopsies after lung transplantation. J Heart Lung Transplant 2008;27: 1128-1134.

6. Estenne M, Maurer JR, Boehler A et al. Bronchiolitis obliterans syndrome 2001: an update of the diagnostic criteria. J Heart Lung Transplant 2002;21: 297-310.

7. Chamberlain D, Maurer J, Chaparro C, Idolor L. Evaluation of transbronchial lung biopsy specimens in the diagnosis of bronchiolitis obliterans after lung transplantation. J Heart Lung Transplant 1994;13: 963-971. 
8. DiGiovine B, Lynch JP, III, Martinez FJ et al. Bronchoalveolar lavage neutrophilia is associated with obliterative bronchiolitis after lung transplantation: role of IL-8. J Immunol 1996;157: 4194-4202.

9. Riise GC, Williams A, Kjellstrom C, Schersten H, Andersson BA, Kelly FJ. Bronchiolitis obliterans syndrome in lung transplant recipients is associated with increased neutrophil activity and decreased antioxidant status in the lung. Eur Respir J 1998;12: 82-88.

10. Henke JA, Golden JA, Yelin EH, Keith FA, Blanc PD. Persistent increases of BAL neutrophils as a predictor of mortality following lung transplant. Chest 1999;115: 403-409.

11. Riise GC, Andersson BA, Kjellstrom C et al. Persistent high BAL fluid granulocyte activation marker levels as early indicators of bronchiolitis obliterans after lung transplant. Eur Respir J 1999;14: 1123-1130.

12. Zheng $\mathrm{L}$, Walters $\mathrm{EH}, \mathrm{Ward} \mathrm{C}$ et al. Airway neutrophilia in stable and bronchiolitis obliterans syndrome patients following lung transplantation. Thorax 2000;55: 53-59.

13. Neurohr C, Huppmann P, Samweber B et al. Prognostic value of bronchoalveolar lavage neutrophilia in stable lung transplant recipients. J Heart Lung Transplant 2009;28: 468-474.

14. Hubner RH, Meffert S, Mundt U et al. Matrix metalloproteinase-9 in bronchiolitis obliterans syndrome after lung transplantation. Eur Respir J 2005;25: 494-501.

15. Behr J, Maier K, Braun B, Schwaiblmair M, Vogelmeier C. Evidence for oxidative stress in bronchiolitis obliterans syndrome after lung and heart-lung transplantation. The Munich Lung Transplant Group. Transplantation 2000;69: 1856-1860. 
16. Gerhardt SG, McDyer JF, Girgis RE, Conte JV, Yang SC, Orens JB. Maintenance azithromycin therapy for bronchiolitis obliterans syndrome: results of a pilot study. Am J Respir Crit Care Med 2003;168: 121-125.

17. Gottlieb J, Szangolies J, Koehnlein T, Golpon H, Simon A, Welte T. Long-term azithromycin for bronchiolitis obliterans syndrome after lung transplantation. Transplantation 2008;85: 36-41.

18. Porhownik NR, Batobara W, Kepron W, Unruh HW, Bshouty Z. Effect of maintenance azithromycin on established bronchiolitis obliterans syndrome in lung transplant patients. Can Respir J 2008;15: 199-202.

19. Verleden GM, Dupont LJ. Azithromycin therapy for patients with bronchiolitis obliterans syndrome after lung transplantation. Transplantation 2004;77: 1465-1467.

20. Yates B, Murphy DM, Forrest IA et al. Azithromycin reverses airflow obstruction in established bronchiolitis obliterans syndrome. Am J Respir Crit Care Med 2005;172: 772-775.

21. Shitrit D, Bendayan D, Gidon S, Saute M, Bakal I, Kramer MR. Long-term azithromycin use for treatment of bronchiolitis obliterans syndrome in lung transplant recipients. J Heart Lung Transplant 2005;24: 1440-1443.

22. Verleden GM, Vanaudenaerde BM, Dupont LJ, Van Raemdonck DE. Azithromycin reduces airway neutrophilia and interleukin-8 in patients with bronchiolitis obliterans syndrome. Am J Respir Crit Care Med 2006;174: 566-570.

23. Vanaudenaerde BM, Meyts I, Vos R et al. A dichotomy in bronchiolitis obliterans syndrome after lung transplantation revealed by azithromycin therapy. Eur Respir J 2008;32: 832-843. 
24. Verleden GM, Vos R, De Vleeschauwer SI et al. Obliterative bronchiolitis following lung transplantation: from old to new concepts? Transpl Int 2009;22: 771-779.

25. Vos R, Vanaudenaerde BM, Verleden SE et al. A randomised controlled trial of azithromycin to prevent chronic rejection after lung transplantation. Eur Respir J 2011;37: 164-172.

26. Verleden GM, Vos R, Verleden SE et al. Survival determinants in lung transplant patients with chronic allograft dysfunction. Transplantation 2011;92: 703-708.

27. Benden C, Boehler A. Long-term clarithromycin therapy in the management of lung transplant recipients. Transplantation 2009;87: 1538-1540.

28. Ibrahim RB, Abella EM, Chandrasekar PH. Tacrolimus-clarithromycin interaction in a patient receiving bone marrow transplantation. Ann Pharmacother 2002;36: 1971-1972.

29. Kunicki PK, Sobieszczanska-Malek M. Pharmacokinetic interaction between tacrolimus and clarithromycin in a heart transplant patient. Ther Drug Monit 2005;27: 107-108.

30. Vos R, Vanaudenaerde BM, Verleden SE et al. Antiinflammatory and Immunomodulatory Properties of Azithromycin Involved in Treatment and Prevention of Chronic Lung Allograft Rejection. Transplantation 2012.

31. Verleden SE, Vos R, Mertens V et al. Heterogeneity of chronic lung allograft dysfunction: insights from protein expression in broncho alveolar lavage. J Heart Lung Transplant 2011;30: 667-673. 
32. D'Ovidio F, Mura M, Tsang M et al. Bile acid aspiration and the development of bronchiolitis obliterans after lung transplantation. J Thorac Cardiovasc Surg 2005;129: 1144-1152.

33. Reynaud-Gaubert M, Marin V, Thirion X et al. Upregulation of chemokines in bronchoalveolar lavage fluid as a predictive marker of post-transplant airway obliteration. J Heart Lung Transplant 2002;21: 721-730.

34. Riise GC, Ericson P, Bozinovski S, Yoshihara S, Anderson GP, Linden A. Increased net gelatinase but not serine protease activity in bronchiolitis obliterans syndrome. J Heart Lung Transplant 2010;29: 800-807.

35. Verleden SE, Vandooren J, Vos R et al. Azithromycin decreases MMP-9 expression in the airways of lung transplant recipients. Transpl Immunol 2011;25: 159-162.

36. Burlingham WJ, Love RB, Jankowska-Gan E et al. IL-17-dependent cellular immunity to collagen type $\mathrm{V}$ predisposes to obliterative bronchiolitis in human lung transplants. J Clin Invest 2007;117: 3498-3506.

37. Fan L, Benson HL, Vittal R et al. Neutralizing IL-17 prevents obliterative bronchiolitis in murine orthotopic lung transplantation. Am J Transplant 2011;11: 911-922.

38. Vanaudenaerde BM, De Vleeschauwer SI, Vos R et al. The role of the IL23/IL17 axis in bronchiolitis obliterans syndrome after lung transplantation. Am J Transplant 2008;8: 19111920.

39. Murphy DM, Forrest IA, Ward C et al. Effect of azithromycin on primary bronchial epithelial cells derived from stable lung allografts. Thorax 2007;62: 834. 
40. Vanaudenaerde BM, Wuyts WA, Geudens $\mathrm{N}$ et al. Macrolides inhibit IL17-induced IL8 and 8isoprostane release from human airway smooth muscle cells. Am J Transplant 2007;7: 76-82.

41. Stewart S, Fishbein MC, Snell GI et al. Revision of the 1996 working formulation for the standardization of nomenclature in the diagnosis of lung rejection. J Heart Lung Transplant 2007;26: 1229-1242.

42. Ross DJ, Marchevsky A, Kramer M, Kass RM. "Refractoriness" of airflow obstruction associated with isolated lymphocytic bronchiolitis/bronchitis in pulmonary allografts. J Heart Lung Transplant 1997;16: 832-838.

43. Glanville AR, Aboyoun CL, Havryk A, Plit M, Rainer S, Malouf MA. Severity of lymphocytic bronchiolitis predicts long-term outcome after lung transplantation. Am J Respir Crit Care Med 2008;177: 1033-1040.

44. De SA, Fisher AJ, Small T, Corris PA. Inhaled corticosteroids and the treatment of lymphocytic bronchiolitis following lung transplantation. Am J Respir Crit Care Med 2001;164: 1209-1212.

45. Chambers DC, Hodge $S$, Hodge $G$ et al. A novel approach to the assessment of lymphocytic bronchiolitis after lung transplantation--transbronchial brush. J Heart Lung Transplant 2011;30: $544-551$.

46. Vos R, Vanaudenaerde BM, Verleden SE et al. Bronchoalveolar lavage neutrophilia in acute lung allograft rejection and lymphocytic bronchiolitis. J Heart Lung Transplant 2010;29: 12591269. 
47. de Jong PA, Dodd JD, Coxson $\mathrm{HO}$ et al. Bronchiolitis obliterans following lung transplantation: early detection using computed tomographic scanning. Thorax 2006;61: 799-804.

48. Chamberlain D, Maurer J, Chaparro C, Idolor L. Evaluation of transbronchial lung biopsy specimens in the diagnosis of bronchiolitis obliterans after lung transplantation. J Heart Lung Transplant 1994;13: 963-971.

49. Sato $M$, Waddell TK, Wagnetz $U$ et al. Restrictive allograft syndrome (RAS): a novel form of chronic lung allograft dysfunction. J Heart Lung Transplant 2011;30: 735-742.

50. Martinu T, Howell DN, Davis RD, Steele MP, Palmer SM. Pathologic correlates of bronchiolitis obliterans syndrome in pulmonary retransplant recipients. Chest 2006;129: 1016-1023.

51. Burton $\mathrm{CM}$, Iversen $\mathrm{M}$, Carlsen J, Andersen $\mathrm{CB}$. Interstitial inflammatory lesions of the pulmonary allograft: a retrospective analysis of 2697 transbronchial biopsies. Transplantation 2008;86: 811-819.

52. Sverzellati N, Zompatori M, Poletti V, Geddes DM, Hansell DM. Small chronic pneumothoraces and pulmonary parenchymal abnormalities after bone marrow transplantation. J Thorac Imaging 2007;22: 230-234.

53. von der Thusen JH, Hansell DM, Tominaga $M$ et al. Pleuroparenchymal fibroelastosis in patients with pulmonary disease secondary to bone marrow transplantation. Mod Pathol 2011;24: 1633-1639.

54. Ofek $E$, Sato $M$, Saito $T$ et al. Restrictive allograft syndrome post lung transplantation is characterized by pleuroparenchymal fibroelastosis. Mod Pathol 2012. 
55. Vos R, Vanaudenaerde BM, De Vleeschauwer SI et al. Follicular bronchiolitis: a rare cause of bronchiolitis obliterans syndrome after lung transplantation: a case report. Am J Transplant 2009;9: 644-650.

56. McManus TE, Milne DG, Whyte KF, Wilsher ML. Exudative bronchiolitis after lung transplantation. J Heart Lung Transplant 2008;27: 276-281.

57. Woodrow JP, Shlobin OA, Barnett SD, Burton N, Nathan SD. Comparison of bronchiolitis obliterans syndrome to other forms of chronic lung allograft dysfunction after lung transplantation. J Heart Lung Transplant 2010;29: 1159-1164.

58. Sato $\mathrm{M}$, Hwang DM, Ohmori-Matsuda $\mathrm{K}$ et al. Revisiting the pathologic finding of diffuse alveolar damage after lung transplantation. J Heart Lung Transplant 2012;31: 354-363.

59. Todd JL, Palmer SM. Bronchiolitis obliterans syndrome: the final frontier for lung transplantation. Chest 2011;140: 502-508.

60. Seibold MA, Wise AL, Speer MC et al. A common MUC5B promoter polymorphism and pulmonary fibrosis. N Engl J Med 2011;364: 1503-1512.

61. Ohshimo S, Bonella F, Sommerwerck U et al. Comparison of serum KL-6 versus bronchoalveolar lavage neutrophilia for the diagnosis of bronchiolitis obliterans in lung transplantation. J Heart Lung Transplant 2011;30: 1374-1380.

62. Kohno N, Kyoizumi S, Awaya Y, Fukuhara H, Yamakido M, Akiyama M. New serum indicator of interstitial pneumonitis activity. Sialylated carbohydrate antigen KL-6. Chest 1989;96: 68-73. 
63. Ohshimo S, Bonella F, Grammann $\mathrm{N}$ et al. Serum KL-6 as a novel disease marker in adolescent and adult cystic fibrosis. Sarcoidosis Vasc Diffuse Lung Dis 2009;26: 47-53.

64. Fisher AJ, Rutherford RM, Bozzino J, Parry G, Dark JH, Corris PA. The safety and efficacy of total lymphoid irradiation in progressive bronchiolitis obliterans syndrome after lung transplantation. Am J Transplant 2005;5: 537-543.

65. Jaksch $P$, Scheed A, Keplinger $M$ et al. A prospective interventional study on the use of extracorporeal photopheresis in patients with bronchiolitis obliterans syndrome after lung transplantation. J Heart Lung Transplant 2012;31: 950-957.

66. Verleden GM, Verleden SE, Vos R et al. Montelukast for bronchiolitis obliterans syndrome after lung transplantation: a pilot study. Transpl Int 2011;24: 651-656.

67. Noble PW, Albera C, Bradford WZ et al. Pirfenidone in patients with idiopathic pulmonary fibrosis (CAPACITY): two randomised trials. Lancet 2011;377: 1760-1769.

68. Nakazato H, Oku H, Yamane S, Tsuruta Y, Suzuki R. A novel anti-fibrotic agent pirfenidone suppresses tumor necrosis factor-alpha at the translational level. Eur J Pharmacol 2002;446: 177-185.

69. Dosanjh A, Ikonen T, Wan B, Morris RE. Pirfenidone: A novel anti-fibrotic agent and progressive chronic allograft rejection. Pulm Pharmacol Ther 2002;15: 433-437.

70. McKane BW, Fernandez F, Narayanan K et al. Pirfenidone inhibits obliterative airway disease in a murine heterotopic tracheal transplant model. Transplantation 2004;77: 664-669. 
71. Zhou H, Latham CW, Zander DS, Margolin SB, Visner GA. Pirfenidone inhibits obliterative airway disease in mouse tracheal allografts. J Heart Lung Transplant 2005;24: 1577-1585.

72. Liu H, Drew $P$, Gaugler AC, Cheng $Y$, Visner GA. Pirfenidone inhibits lung allograft fibrosis through L-arginine-arginase pathway. Am J Transplant 2005;5: 1256-1263.

73. Reams BD, Davis RD, Curl J, Palmer SM. Treatment of refractory acute rejection in a lung transplant recipient with campath $1 \mathrm{H}$. Transplantation 2002;74: 903-904.

74. Reams BD, Musselwhite LW, Zaas DW et al. Alemtuzumab in the treatment of refractory acute rejection and bronchiolitis obliterans syndrome after human lung transplantation. Am J Transplant 2007;7: 2802-2808.

75. Ruiz-Arguelles GJ, Ruiz-Delgado GJ, Moreno-Ford V. Re: Alemtuzumab-induced resolution of pulmonary noninfectious complications in a patient with chronic graft-versus-host disease. Biol Blood Marrow Transplant 2008;14: 1434-1435.

76. Kohno M, Perch M, Andersen E, Carlsen J, Andersen CB, Iversen M. Treatment of intractable interstitial lung injury with alemtuzumab after lung transplantation. Transplant Proc 2011;43: 1868-1870. 